\title{
USEFULNESS OF CD44 LEVEL AS A BIOMARKER IN DIAGNOSIS OF ACUTE LIVER REJECTION
}

\author{
By
}

ESAM M. BAYOMY ${ }^{1}$, TAREK M. YOUSEF ${ }^{1}$, KHALID M. MANSOUR ${ }^{1}$, DOAA Z. ZAKY ${ }^{2}$, AHMED M. EL GHANDOUR ${ }^{1}$ AND MOHAMED B. AHMED ${ }^{3}$ Department of Internal Medicine and Gastroentrology ${ }^{1}$, Department of Tropical Medicine $^{2}$ and Department of General Surgery and Liver Transplantation, Faculty of Medicine, Ain Shams University, Cairo 11566, Egypt

( ${ }^{\star}$ Correspondence: drdoaazakaria@gmail.com)

\begin{abstract}
The incidence of acute and chronic rejection has declined with improvement of immunosuppression regimens in liver transplant recipients. The CD44 protein belongs to a large family of type I Trans membrane glycoproteins and expressed on the surface of most vertebrate cells and is an important receptor for the components of extracellular matrix.

The study evaluated if the serum level of CD44 had a value in diagnosis of acute rejection depending on proved rejection by liver biopsy. The patients were recruited from ASCOT from May 2017 to December 2018. They were 20 adults with Post LDLT with elevated liver function tests and were reviewed.

The results showed that serum level of CD44 was significantly lower in the rejection group in our study in patient with post liver transplantation. There was highly significant difference between controls \& patients las regards serum level of CD44. Negative correlation between serum levels of CD44, WBCs \& AST. There was highly significant difference between patients with different pathological findings as regards serum level of CD44. The best cut off point for serum level of CD44 as a marker of rejection was found $\leq 44 \mathrm{ng} / \mathrm{ml}$ with sensitivity of $85 \%$ and specificity of $95 \%$ in diagnosis of acute cellular rejection.
\end{abstract}

Key words: Egyptian patients, Liver transplantation, CD44, Transplant immunology.

\section{Introduction}

Liver transplantation (LT) is considered as an established therapeutic option for patients with the acute and chronic liver failure and hepatocellular carcinoma (Graziadei et al, 2016). It evolved as a highly effective approach to treat many end-stage liver disease (ESLD) cases that had had no treatment before LT (Sagmeister et al, 2002). Patients with liver cirrhosis were more susceptible to infections, due to the alterations in the gut microbiota, intestinal barrier dysfunction, genetic predisposition, and immunodysfunction (Jalan et al, 2012). These immunocompromised patients are predisposed to develop bacterial infections and sepsis, due to endothelial alterations, leukocyte dysfunction, bacterial translocation, and iatrogenic factors (Acevedo and Fernandez, 2014). Moreover, the acute-on-chronic liver failure may develop as sequela of a superimposed bacterial infection or sepsis (Moreau et al, 2013).

Generally, many Egyptian authors declared that the Egyptian patients suffered from the increasing number of chronic LDs and LDs, due to the high prevalence of hepatitis $\mathrm{C}$ virus among the population, with an increasing need for LT (Sharaf-Eldin et al, 2016; Ahmad et al, 2017; 2018; Abd-Elsalam et al, 2018).

The first human orthotropic liver transplantation (LT) in Europe was performed by Sir Roy Calne in Cambridge in 1968 (Calne et al, 1968). One year after the first successful human liver transplantation reported by Thomas Starzl in the United States (Starzl et $a l$, 1967). The LT has been evolved rapidly, as standard therapy for acute and chronic liver failure of all etiologies, with more than 80,000 procedures performed to date. Survival rates were improved significant in the last two decades, achieved rates of $96 \%$ \& $71 \%$ at $1 \& 10$ years post-LT respectively (Adam et al, 2012). This great success was mostly attributable to several advances such 
as the introduction of new immunosuppressive agents and preservation solutions, to the improvements in surgical techniques and to early diagnosis and management of complications after LT (Dutkowski et al, 2010). Despite the continuous optimization of immunosuppression protocols, the acute cellular rejection (ACR) occurred in about $15 \%$ $60 \%$ of liver allograft recipients in the first postoperative months (Rostaing et al, 2012).

In contrast to many aspects of clinical transplantation, the algorithm for diagnosing ACR has not changed since the advent of clinical transplantation in the 1960s. The diagnosis of ACR requires evidence of graft dysfunction (e.g., elevated aminotransferases), which was typically followed by confirmed allograft biopsy. Liver biopsy was the best organ for ACR diagnosis (Demetris et al, 2010).

The postoperative monitoring of liver transplant recipients in regard to ACR was based on measurements of transaminase levels and markers of bile synthesis and accumulation. In cases of parameters increase, a biopsy was indicated for histopathological validation and differentiation from other reasons for graft dysfunction, such as ischemia/reperfusion injury or recurrent hepatitis (Rook and Rand, 2011).

Biomarkers for noninvasive diagnosis and, ideally, for the prediction of ACR, would be a valuable tool for the postoperative care of liver transplant recipients. Such biomarkers could facilitate a considerably earlier diagnosis and enable individual risk stratification and the adjustment of immunosuppressive regimens to prevent rejection (Roedder et al, 2011).

When a foreign graft is inserted into a recipient, recipient leukocytes respond to the foreign antigens by producing soluble factors that induce both lymphocyte and antigen proliferation. Thus, cytokines as interleukins (IL) IL-1, IL-2, IL-5, IL-6, tumor necrosis factor (TNF), and g-interferon (gIFN) may be expected to rise in response to an episode of acute rejection. Substances such as b2-microglobulin (b2-M), intercellular adhesion molecule- 1 (ICAM-1) and ne- opterin, which were induced by cytokines, may also be expected to be of potential use. However, secretion of these substances is a reflection of leukocyte activation and is not specific for rejection. The IL-1, TNF, \& gIFN measurement proved to be disappointing, with increases in all agents in rejection, infection, and other complications (Tilg et al, 1990).

The CD44 can be used as a biomarker for the blood test-based diagnosis of rejection in liver transplant recipients. These results may help us identify patients who are at risk for ACR in the first months after transplantation to improve the clinical care of liver transplant recipients (Raschzok et al, 2015).

CD44 is a family of type I transmembrane glycoproteins with a wide tissue distribution that is involved in various physiological and pathological processes, such as cell-cell and cell-matrix interaction, leukocyte extravasation, cell migration, and lymphocyte activation (Yan et al, 2015). Serum soluble CD44 can be generated through the proteolytic cleavage of membrane- or receptor-bound CD44 or by cell activation, for example, in autoimmune disorders (Katoh et al, 1994). Also, the involvement of CD44 in inflammatory $\mathrm{T}$ helper 1 immune responses, its role in regulatory $\mathrm{T}$ cell function (Sharma, 2017).

The study aimed to assess value of serum level of CD44 as a marker of rejection in patients with post liver transplantation

\section{Materials and Methods}

This study included two groups of Egyptian patients selected from Ain Shams Center of Organ Transplantation (ASCOT). Patients were recruited from May 2017 to December 2018. They were divided into two groups: G1 (patients): Twenty post Living Donor Liver Transplantation (LDLT) patients with elevated liver enzymes within first three months post-operative. G2 (control): Twenty persons with normal liver profile and free from any diseases (Donors preoperative were included). The study was explained, and written consents were taken from all patients. Patients with the following 
criteria were excluded: 1- Patients with Infection excluded with CRP, Procalcitonin, culture and sensitivity of body fluids and secretions, 2- Patients with Vascular complication excluded with Hepatic Venous and Arterial Duplex, and 3- Patients with surgical biliary tract complications.

Patients assessments: History taking with special emphasis on: All patients were subjected to: age, sex, fever, date of surgery (LDLT), drug history, drug history of immunosuppression, complications and sympto$\mathrm{ms}$ of hepatic decompensating pre-operative e.g. hematemesis, ascites, hepatic encephalopath....etc. Itching, abdominal pain, vomiting. Full physical examination included abdominal examination with special emphasis on jaundice, drains and wound.

Biochemical analysis: The following tests were done: Complete blood count (CBC), liver profile tests, alanine transaminase (ALT), aspartate transaminase (AST), alkaline Phosphatase, GGT, serum Bilirubin, serum Albumin and prothrombin time (PT). CRP, Procalcitonin, virology tests e.g. HCV RNA PCR, CMV PCR, EBV IgM, HBV DNA PCR, Culture and sensitivity of body fluids, secretions, serum level of immunosuppression drug and serum CD44 level.

Imaging: Ultrasound, hepatic venous and arterial duplex were done.

The liver biopsy and histopathological examination: ACR changes were divided into 3 pathological pictures; mixed (predominantly mononuclear activated lymphocytes, neutrophils \& eosinophils) portal inflammation, bile duct inflammation/damage and subendothelial inflammation of portal veins or terminal hepatic venules (Choudhary et al, 2017).

Each of these parameters was scored as 1 to 3 and sum was called rejection activity index, thus a maximum score of 9 was possible. The various possible rejection grades were: a score of $0-2$ without rejection, 3 borderlines (with consistent), 4-5 mild, 6-7 moderate and 8-9 as severe ACR. Higher rejection activity index did not translate into less response to steroid (Höroldt et al, 2006). CD44 serum level assay2.2.5: After collection of blood, blood was clot by leaving it undisturbed at room temperature, 1020 minutes. Remove clot by centrifuging at 2,000-3,000rpm for 20 minutes. If precipitates appeared during reservation, the sample must be centrifuged again.

Statistical analysis: Data were analyzed by statistical package (SPSS) software version 13.0. Data were expressed as number and percentage and continuous data as in laboratory data as mean and SD. If data was normally distrusted Chi-square test $\left(\mathrm{x}^{2}\right)$ (test of significance in categorical data). Paired $\mathrm{T}$ test was used for comparing between 2 dependent means. If data was not normally disturbed Mann Whitney U test for two-group comparisons was used. $\mathrm{P}$ value above 0.05 was significant.

Ethics and consent: The participants provided written informed consent, and the study was approved by the Ain Shams University Faculty of Medicine Research Ethical Committee.

\section{Results}

The results were shown in tables $(1,2,3,4$, $5,6,7,8 \& 9)$

Table 1: Comparison between Age, Sex, Hx of HCC, Co-morbidity and Fever among controls

\begin{tabular}{|l|l|c|c|c|c|}
\hline \multicolumn{2}{|l|}{ Variants } & Control $(\mathrm{n}=20)$ & Patients $(\mathrm{n}=20)$ & P-value & Significant \\
\hline & $\mathrm{M} \pm \mathrm{SD}$ & $29.80 \pm 4.25$ & $42.60 \pm 12.73$ & & \\
Age & Range & $26-41$ & $16-59$ & 0.000 & HS \\
\hline \multirow{3}{*}{ sex } & Male & $14(70.0 \%)$ & $14(70.0 \%)$ & & \\
\hline & Female & $6(30.0 \%)$ & $6(30.0 \%)$ & 1.000 & NS \\
\hline Co-morbidities & No & $20(100.0 \%)$ & $12(60.0 \%)$ & & \\
\hline & DM & $0(0.0 \%)$ & $2(10.0 \%)$ & 0.007 & HS \\
\hline \multirow{3}{*}{ Fever } & DM Hypothyroidism & $0(0.0 \%)$ & $6(30.0 \%)$ & & \\
\hline
\end{tabular}

*:Chi-square test; $\bullet$ : Independent t-test 
Table 2: Comparison between different labs (CBC, CRP, INR, PTT, Bili, AST, ALT) among controls \& patients.

\begin{tabular}{|c|c|c|c|c|c|c|}
\hline \multicolumn{2}{|l|}{ Variables } & Control $(n=20)$ & Patients $(n=20)$ & Test value & $\mathrm{P}$-value & Significant \\
\hline $\mathrm{Hb}(\mathrm{gm} / \mathrm{d})$ & $\begin{array}{l}\mathrm{M} \pm \mathrm{SD} \\
\text { Range }\end{array}$ & $\begin{array}{c}13.36 \pm 1.27 \\
11.1-15.2\end{array}$ & $\begin{array}{c}8.73 \pm 0.99 \\
7.1-10.6\end{array}$ & $12.877 *$ & 0.000 & HS \\
\hline Plt $\left(\times 10^{3} / \mathrm{ml}\right)$ & $\begin{array}{l}\mathrm{M} \pm \mathrm{SD} \\
\text { Range }\end{array}$ & $\begin{array}{c}225.55 \pm 88.47 \\
188-325\end{array}$ & $\begin{array}{c}127.40 \pm 92.61 \\
32-308\end{array}$ & $3.427 *$ & 0.001 & HS \\
\hline $\mathrm{WBCs}\left(\times 10^{3} / \mathrm{ml}\right)$ & $\begin{array}{l}\mathrm{M} \pm \mathrm{SD} \\
\text { Range }\end{array}$ & $\begin{array}{c}6.20 \pm 1.18 \\
4.5-8.5\end{array}$ & $\begin{array}{c}7.62 \pm 5.22 \\
1.8-17.9 \\
\end{array}$ & $-1.186^{*}$ & 0.243 & NS \\
\hline $\mathrm{CRP}(\mathrm{mg} / \mathrm{L})$ & $\begin{array}{l}\mathrm{M} \pm \mathrm{SD} \\
\text { Range }\end{array}$ & $\begin{array}{c}0.26 \pm 0.16 \\
0-0.5\end{array}$ & $\begin{array}{l}2.86 \pm 3.80 \\
0.07-12.2 \\
\end{array}$ & $-3.058^{*}$ & 0.004 & HS \\
\hline INR & $\begin{array}{l}\mathrm{M} \pm \mathrm{SD} \\
\text { Range }\end{array}$ & $\begin{array}{c}1.05 \pm 0.07 \\
0.9-1.1\end{array}$ & $\begin{array}{c}1.27 \pm 0.29 \\
1-2\end{array}$ & $-3.278^{*}$ & 0.002 & HS \\
\hline PTT (sec) & $\begin{array}{l}\mathrm{M} \pm \mathrm{SD} \\
\text { Range }\end{array}$ & $\begin{array}{c}33.66 \pm 5.36 \\
21.9-43\end{array}$ & $\begin{array}{c}37.85 \pm 5.77 \\
31-50.3\end{array}$ & $-2.380^{*}$ & 0.022 & $\mathrm{~S}$ \\
\hline T. Bili ( mg/dl) & $\begin{array}{l}\mathrm{M} \pm \mathrm{SD} \\
\text { Range }\end{array}$ & $\begin{array}{c}0.52 \pm 0.15 \\
0.3-0.7 \\
\end{array}$ & $\begin{array}{c}11.86 \pm 9.80 \\
1.2-25.9\end{array}$ & $-5.171 *$ & 0.000 & HS \\
\hline D. Bili $(\mathrm{mg} / \mathrm{dl})$ & $\begin{array}{l}\mathrm{M} \pm \mathrm{SD} \\
\text { Range }\end{array}$ & $\begin{array}{c}0.17 \pm 0.08 \\
0.1-0.3\end{array}$ & $\begin{array}{c}6.85 \pm 5.31 \\
0.3-13.7\end{array}$ & $-5.626^{*}$ & 0.000 & HS \\
\hline AST (U/L) & $\begin{array}{l}\text { Median (IQR) } \\
\text { Range }\end{array}$ & $\begin{array}{c}17(16-20) \\
14-28\end{array}$ & $\begin{array}{c}59.5(51-94) \\
36-244\end{array}$ & $-5.423 \neq$ & 0.000 & HS \\
\hline ALT (U/L) & $\begin{array}{c}\text { Median(IQR) } \\
\text { Range }\end{array}$ & $\begin{array}{c}15(1-16) \\
11-18 \\
\end{array}$ & $\begin{array}{c}82(71-136) \\
49-638\end{array}$ & $-5.421 \neq$ & 0.000 & HS \\
\hline
\end{tabular}

Table 3: Comparison between ALK, GGT, T. Prot, S.Alb, BUN, S.Creat, Na, K, P.Cal in controls \& patients.

\begin{tabular}{|c|c|c|c|c|c|c|}
\hline \multicolumn{2}{|l|}{ Variables } & Control $(n=20)$ & Patients $(n=20)$ & Test value & P-value & Significant \\
\hline ALK P (U/L) & $\begin{array}{l}\mathrm{M} \pm \mathrm{SD} \\
\text { Range }\end{array}$ & $\begin{array}{c}80.00 \pm 21.72 \\
42-120\end{array}$ & $\begin{array}{c}400.80 \pm 280.56 \\
126-909\end{array}$ & $-5.098^{*}$ & 0.000 & HS \\
\hline GGT (U/L) & $\begin{array}{l}\mathrm{M} \pm \mathrm{SD} \\
\text { Range }\end{array}$ & $\begin{array}{c}30.00 \pm 12.19 \\
12-48\end{array}$ & $\begin{array}{c}378.80 \pm 256.35 \\
44-866\end{array}$ & $-6.078 *$ & 0.000 & HS \\
\hline T. Prot (g/dl) & $\begin{array}{l}\text { Median(IQR) } \\
\text { Range }\end{array}$ & $\begin{array}{c}7.2(6.9-7.8) \\
5.9-71\end{array}$ & $\begin{array}{c}5.8(5.5-6.6) \\
3.8-7.6\end{array}$ & $-3.957 \neq$ & 0.000 & HS \\
\hline S. Alb (g/dl) & $\begin{array}{l}\mathrm{M} \pm \mathrm{SD} \\
\text { Range }\end{array}$ & $\begin{array}{c}4.32 \pm 0.56 \\
3.5-5.2\end{array}$ & $\begin{array}{c}2.75 \pm 0.67 \\
2-4.4\end{array}$ & $8.065^{*}$ & 0.000 & HS \\
\hline BUN (mg/dl) & $\begin{array}{l}\mathrm{M} \pm \mathrm{SD} \\
\text { Range }\end{array}$ & $\begin{array}{c}14.60 \pm 2.98 \\
10-19\end{array}$ & $\begin{array}{c}38.50 \pm 26.58 \\
11-104\end{array}$ & $-3.996^{*}$ & 0.000 & HS \\
\hline S. Creat $(\mathrm{mg} / \mathrm{d})$ & $\begin{array}{l}\mathrm{M} \pm \mathrm{SD} \\
\text { Range }\end{array}$ & $\begin{array}{c}0.76 \pm 0.20 \\
0.5-1.2\end{array}$ & $\begin{array}{c}1.12 \pm 0.41 \\
0.6-1.9\end{array}$ & $-3.527 *$ & 0.001 & HS \\
\hline $\mathrm{Na}(\mathrm{m} \mathrm{Eq} / \mathrm{L})$ & $\begin{array}{l}\mathrm{M} \pm \mathrm{SD} \\
\text { Range }\end{array}$ & $\begin{array}{c}141.20 \pm 2.42 \\
136-145\end{array}$ & $\begin{array}{c}132.0 \pm 4.83 \\
122-139\end{array}$ & $7.611^{*}$ & 0.000 & HS \\
\hline $\mathrm{K}(\mathrm{m} \mathrm{Eq} / \mathrm{L})$ & $\begin{array}{l}\mathrm{M} \pm \mathrm{SD} \\
\text { Range }\end{array}$ & $\begin{array}{l}4.34 \pm 0.38 \\
3.8-5\end{array}$ & $\begin{array}{c}3.95 \pm 0.74 \\
2.3-4.9\end{array}$ & $2.094^{*}$ & 0.043 & $\mathrm{~S}$ \\
\hline P. Cal (ng/ml) & $\begin{array}{l}\mathrm{M} \pm \mathrm{SD} \\
\text { Range }\end{array}$ & $\begin{array}{c}0.18 \pm 0.12 \\
0-0.4\end{array}$ & $\begin{array}{c}0.31 \pm 0.13 \\
0.1-0.5\end{array}$ & $-3.547^{*}$ & 0.001 & HS \\
\hline
\end{tabular}

No significant difference between controls $\&$ patients as regards $\mathrm{WBC}$ but, significant difference as regards PTT with $(\mathrm{P}=0.022$ and highly significant difference as regard $\mathrm{Hb}(\mathrm{P}=0.000)$, Plt $(\mathrm{P}=0.001), \mathrm{CRP}(\mathrm{P}=$ 0.004), INR ( $\mathrm{P}=0.002)$, T. Bili $(\mathrm{P}=0.000)$, D. Bili $(P=0.000)$ AST $(P=0.000)$, ALT
$(P=0.000)$. There was highly significant difference between controls \& patients las regards ALK $(\mathrm{P}=0.000)$, GGT $(\mathrm{P}=0.000)$, T. protein $(\mathrm{P}=0.000)$, S.Alb $(\mathrm{P}=0.000)$, BUN $(\mathrm{P}=0.000), \mathrm{S}$. ceat. $(\mathrm{P}=0.001), \mathrm{Na}(\mathrm{P}=$ $0.000)$, $\mathrm{PCal}(\mathrm{P}=0.001)$ but significant difference as regards $K(P=0.043)$.

Table 4: Comparison between serum level of CD44 among controls \& patients.

\begin{tabular}{|l|c|c|c|c|c|}
\hline CD44 & Control $(\mathrm{n}=20)$ & Patients $(\mathrm{n}=20)$ & Test value & P-value & Significant \\
\hline Median(IQR) & $61.25(51.25 \pm 90)$ & $14.5(13 \pm 40.25)$ & & & \\
Range $(\mathrm{ng} / \mathrm{ml})$ & $35-120$ & $6.5-75$ & -4.460 & 0.000 & HS \\
\hline
\end{tabular}


There was highly significant difference between controls \& patients as regards se-

Table 5: Comparison between PA U/S parameters and biopsy among controls \& patients.

\begin{tabular}{|c|c|c|c|c|c|c|}
\hline \multicolumn{2}{|l|}{ Variables } & Control $(n=20)$ & Patients $(n=20)$ & Test value & P-value & Significant \\
\hline P.V PSV & $\begin{array}{l}\mathrm{M} \pm \mathrm{SD} \\
\text { Range }\end{array}$ & $\begin{array}{c}35.00 \pm 1.72 \\
32-37\end{array}$ & $\begin{array}{c}56.05 \pm 12.79 \\
38.5-86\end{array}$ & $-7.294 \bullet$ & 0.000 & HS \\
\hline H.A RI & $\begin{array}{l}\mathrm{M} \pm \mathrm{SD} \\
\text { Range }\end{array}$ & $\begin{array}{c}0.57 \pm 0.18 \\
0.07-0.69\end{array}$ & $\begin{array}{c}0.56 \pm 0.18 \\
0.06-0.71\end{array}$ & $0.292 \bullet$ & 0.772 & NS \\
\hline Hepatic vein & Triphasic & $20(100.0 \%)$ & $20(100.0 \%)$ & $0.000 *$ & 1.000 & NS \\
\hline Collection & $\begin{array}{l}\text { No } \\
\text { Yes }\end{array}$ & $\begin{array}{c}20(100.0 \%) \\
0(0.0 \%)\end{array}$ & $\begin{array}{c}18(90.0 \%) \\
2(10.0 \%)\end{array}$ & $2.105^{*}$ & 0.147 & NS \\
\hline \multirow[t]{4}{*}{ Liver Biopsy } & Rejection & $0(0.0 \%)$ & $14(70.0 \%)$ & & & \\
\hline & +vascular insult Cholangitis + & \multirow{3}{*}{$\begin{array}{c}0(0.0 \%) \\
0(0.0 \%) \\
20(100.0 \%) \\
\end{array}$} & $2(10.0 \%)$ & \multirow[t]{3}{*}{$40.000^{*}$} & \multirow[t]{3}{*}{0.000} & \multirow[t]{3}{*}{ HS } \\
\hline & Obs & & $4(20.0 \%)$ & & & \\
\hline & Normal & & $0(0.0 \%)$ & & & \\
\hline
\end{tabular}

*:Chi-square test; $\bullet$ : Independent t-test

Non-significant difference was between controls \& patients as regards H.A R.I, Hepatic vein and collection, But, a highly signif- rum level of $\mathrm{CD} 44$ with $(\mathrm{P}=0.000)$ high with controls and low with patients.

Table 6: Correlation between variable parameters and serum level of CD44 in patients

\begin{tabular}{|l|c|c|}
\hline Variables & $\mathrm{r}$ & P-value \\
\hline Age & 0.294 & 0.209 \\
\hline Hb & -0.110 & 0.643 \\
\hline Plt & $-0.628^{* * *}$ & 0.003 \\
\hline WBCs & $-0.572^{* *}$ & 0.008 \\
\hline CRP & -0.109 & 0.648 \\
\hline INR & -0.073 & 0.760 \\
\hline PTT & -0.104 & 0.663 \\
\hline T.Bili & -0.048 & 0.842 \\
\hline D.Bili & -0.097 & 0.686 \\
\hline AST & $-0.483^{*}$ & 0.031 \\
\hline ALT & -0.222 & 0.346 \\
\hline ALK P & -0.239 & 0.310 \\
\hline GGT & -0.322 & 0.166 \\
\hline T.Prot & 0.316 & 0.175 \\
\hline s.Alb & -0.057 & 0.812 \\
\hline BUN & 0.035 & 0.882 \\
\hline S.Creat & -0.196 & 0.408 \\
\hline Na & $0.722^{* * *}$ & 0.000 \\
\hline K & -0.215 & 0.362 \\
\hline P.Cal & 0.011 & 0.963 \\
\hline Level of FK & -0.351 & 0.263 \\
\hline Level of Neoral & 0.395 & 0.333 \\
\hline P.V PSV & 0.172 & 0.469 \\
\hline H.A RI & -0.173 & 0.466 \\
\hline
\end{tabular}

Table 7: Comparison between CD44 levels among patients with different pathological findings in liver biopsy.

\begin{tabular}{|c|c|c|c|c|c|c|c|}
\hline CD44 & Rejection $(=14)$ & $\begin{array}{l}\text { Rejection+ vascular } \\
\text { insult }(n=2)\end{array}$ & $\begin{array}{c}\text { Cholangitis }+ \text { Obs } \\
(\mathrm{n}=4)\end{array}$ & $\begin{array}{l}\text { Controls } \\
(n=20)\end{array}$ & $\begin{array}{l}\text { Test } \\
\text { value }\end{array}$ & $\begin{array}{c}\mathrm{P}- \\
\text { value }\end{array}$ & $\begin{array}{l}\text { Signi- } \\
\text { ficant }\end{array}$ \\
\hline Median(IQR) & $13.00(13.0-14.5)$ & $72.50(70.0-75.0)$ & $41.00(36.5-48.5)$ & $61.25(51.25-90)$ & & & \\
\hline \multirow[t]{3}{*}{ Range (ng/ml) } & $6.5-42.5$ & $70-75$ & \begin{tabular}{|l|}
$35-53$ \\
\end{tabular} & $35-120$ & 28.841 & 0.000 & HS \\
\hline & \multicolumn{7}{|c|}{ Post Hoc analysis by LSD } \\
\hline & P1 & $\mathrm{P} 2$ & \begin{tabular}{|r|} 
P3 \\
\end{tabular} & $\mathrm{P} 4$ & P5 & \multicolumn{2}{|c|}{ P6 } \\
\hline CD44 & 0.022 & 0.005 & 0.064 & 0.000 & 0.042 & \multicolumn{2}{|c|}{0.018} \\
\hline
\end{tabular}

$\$=$ Kruskal Wallis test, P1: Rejection Vs Rejection+ vascular insult P2: Rejection Vs Cholangitis + Obs,P3: Rejection + vascular insult vs cholangitis + obs , p4: control vs rejection ,p5: control vs rejection+ vascular insult and P6: control vs cholangitis + Obs 
Table 8: Comparison between sex, comorbidities, fever, immunosuppression drug, collection in PA U/S, liver biopsy, and serum level of CD44.

\begin{tabular}{|c|c|c|c|c|c|c|}
\hline \multicolumn{2}{|l|}{ Variables } & CD44 Median(IQR) & Range & Test value & P-value & Significant \\
\hline Sex & $\begin{array}{l}\text { Male } \\
\text { Female }\end{array}$ & $\begin{array}{c}14.25(13.00-32.50) \\
41.00(6.50-70.00)\end{array}$ & $\begin{array}{c}13.00-53.00 \\
6.50-75.00 \\
\end{array}$ & $-0.921 \neq$ & 0.357 & NS \\
\hline & No & $14.50(13.00-37.50)$ & $13.00-75.00$ & & & \\
\hline Co. Morbidities & $\mathrm{Dm}$ & $44.00(35.00-53.00)$ & $35.00-53.00$ & 3.289\# & 0.193 & NS \\
\hline & Hypothyroidism & $13.00(6.50-38.00)$ & $6.50-44.00$ & & & \\
\hline Fever & $\begin{array}{l}\text { No } \\
\text { Yes }\end{array}$ & $\begin{array}{l}13.00(13.00-13.75) \\
23.50(13.00-43.25)\end{array}$ & $\begin{array}{c}13.00-14.50 \\
6.50-75.00 \\
\end{array}$ & $-1.391 \neq$ & 0.164 & NS \\
\hline Drug & $\begin{array}{l}\text { F.K } \\
\text { Neoral }\end{array}$ & $\begin{array}{c}24.75(13.00-48.50) \\
13.50(9.75-23.50)\end{array}$ & $\begin{array}{c}13.00-75.00 \\
6.50-42.50\end{array}$ & $-1.645 \ddagger$ & 0.100 & NS \\
\hline Collection & $\begin{array}{l}\text { No } \\
\text { Yes } \\
\end{array}$ & $\begin{array}{l}14.25(13.00-35.00) \\
41.00(38.00-44.00)\end{array}$ & \begin{tabular}{|c|}
$6.50-75.00$ \\
$38.00-44.00$ \\
\end{tabular} & $-1.407 \ddagger$ & 0.159 & NS \\
\hline & Rejection & $13.00(13.00-14.50)$ & $6.50-42.50$ & & & \\
\hline Liver Biopsy & Rejection+ vascular insult & $72.50(70.00-75.00)$ & $70.00-75.00$ & 11.703\# & 0.003 & $\mathrm{~S}$ \\
\hline & Cholangitis + Obs & $41.00(36.50-48.50)$ & $35.00-53.00$ & & & \\
\hline
\end{tabular}

Table 9: Sensitivity \& specificity of CD44 serum level as a marker for acute cellular rejection in post-liver transplantation

\begin{tabular}{|l|c|c|c|c|c|c|}
\hline Parameter & AUC & Cut of Point & Sensitivity & Specificity & PPV & NPV \\
\hline CD44 & 0.911 & $\leq 44$ & 85.00 & 95.00 & 94.4 & 86.4 \\
\hline \multicolumn{7}{|c|}{ AUC: Area under Curve }
\end{tabular}

Best cut off point for of CD44 serum level as a marker of rejection was $\leq 44 \mathrm{ng} / \mathrm{ml}$ with sensitivity of $85 \%$ and specificity of $95 \%$ in diagnosis of acute cellular rejection.

\section{Discussion}

The identification and clinical establishment of easily obtainable and reliable noninvasive biomarkers for ACR would mean substantial progress for postoperative management of the liver transplant recipients (Raschzok et al, 2015).

Biomarkers for the noninvasive diagnosis of ACR may prevent delays in therapy because of sampling or interpretation error, and biopsy-associated complications such as bleeding, infection, or pain (Lee, 2014).

Biomarkers for the stratification of the risk of ACR could help to prevent rejection and individualize the immunosuppression therapy. Noninvasive biomarkers could enable personalized and optimized therapy for liver transplant recipients in an attempt to prevent morbidity and improve allograft survival, thereby reducing hospital stay and limiting the expense of the treatment (Morris and Anderson, 2013).

The aim of this study was to evaluate the significance of serum level of CD44 as a
Marker of acute cellular rejection in patients with post liver transplantation.

The strength of the current study was that the subjects included in our study were with abnormal liver profile all of them underwent liver biopsy to investigate underlying pathology and all of them were examined with one single histopathologist and all laboratory investigations were done in the same laboratory. In our study we depended on diagnosis of acute cellular rejection on histopathological findings in liver biopsy. All patients group were selected from ASCOT after monitoring elevation of the liver functions tests. All controls were selected completely clinically and lab normal persons. Our reference range could be calculated by using the mean of serum level of CD44 of control group (being $=61.25(51.25-90)$.

Chen et al. (2010) described CD31, CD44, and chemokine (C-X-C motif) ligand (CXCL) 9, biomarkers for cross-organ allograft rejection. Rouschop et al. (2010) observed high CD44 protein levels during renal allograft rejection in a study with 23 patients with biopsy-proven acute renal allograft rejection compared with 9 transplant recipients without ACR. Moreover, they reported increased CD44 serum protein levels 
in 24-hour pretransplant blood analyses of renal transplant recipients who later developed acute allograft rejection (Raschzok et al, 2015).

In the present study, serum level of CD44 was significantly lower in the rejection group of patient with post liver transplantation, whereas it was reported to be increased during allograft rejection in the post cardiac and kidney transplantation. The interestingly, through the differentiation of CD4- and CD8-positive lymphocytes in the pretransplant analysis, there is lower CD44 expression for CD8-positive cells and a similar trend for CD4-positive cells in the rejection group. Raschzok et al. (2015) speculated on the reasons for the discrepancy between our findings of low CD44 serum protein levels in liver transplant recipients prior and during rejection and the previously reported high CD44 levels during cardiac and kidney allograft rejection.

In the present study, there was highly significant difference between control group \& patients group as regards serum level of CD44. Negative correlation between serum level of CD44, WBCs and AST .There was highly significant difference between patients with different pathological findings as regards serum level of CD44. The best cut off point for Serum level of CD44 as a marker of rejection was found $\leq 44 \mathrm{ng} / \mathrm{ml}$ with sensitivity of $85 \%$ and specificity of $95 \%$ in diagnosis of acute cellular rejection.

In a cohort study, serum samples were collected in standard serum tubes immediately before transplantation, at POD 1, 3, 7, \& 14, and when a biopsy was performed all patients underwent follow up serial serum levels of CD44 \& CXCL9 then the comparison between serum levels of CD44 \& CXCL9 at different times and pathological findings in liver biopsy (Raschzok et al, 2015).

In the present study, on the basis of a ROC curve analysis, cutoffs were calculated to define the levels of CD44 that differentiate with the highest specificity and sensitivity between patients with and without risk for
ACR. At POD 1, the cutoff values of CD44 for the risk of ACR were identified to be $<200.5 \mathrm{ng} / \mathrm{mL}$ (sensitivity, $88 \%$; specificity, $61 \%$ ), whereas the cutoff values of CXCL9 were $>2.7 \mathrm{ng} / \mathrm{mL}$ (sensitivity, $60 \%$; specificity, 79\%). A combination of both biomarker cutoffs at POD 1 (<CD44 \& >CXCL9 cutoff) enabled the best prediction of patients at risk for ACR with a positive predictive value (PPV) of $91 \%$ and a negative predictive value (NPV) of 67\%, (Asaoka et al, 2014).

In the study of Raschzok et al. (2015) the reference range was different from the present study due to different kits. Massoud et al. (2010) postulated that C4 With a cutoff value of $\leq 0.31 \mathrm{~g} / \mathrm{L}, \mathrm{C} 4$ had a sensitivity of $97 \%$, a specificity of $62 \%$, a positive predictive value of $74 \%$, and a negative predictive value of $94 \%$ as a noninvasive marker of acute cellular rejection in post liver transplant. Feussner et al. (1994) postulated that the mean postoperative SAA plasma concentration in liver allograft recipients was about ten times higher $(9.76 \pm 6.60 \mathrm{mg} / \mathrm{dl})$ as compared to mean value in healthy controls $(0.98 \pm 0.42 \mathrm{mg} / \mathrm{dl})$.

Brouard and Soulillou (2010) evaluated the intracellular IL-2 quantification in $\mathrm{CD} 3+\mathrm{CD} 8+$ cells in 21 liver transplant recipients for 6 months after liver transplantation, showing that intracellular IL-2 expression in CD8+ $\mathrm{T}$ cells before transplantation was closely related to the development of ACR. These results were confirmed by Germani et al. (2009) reported that patients experiencing ACR showed a significantly higher intracellular percentage of IL-2+ in CD8+ T cells compared to stable liver transplant recipients. Graft eosinophilia was identified as an independently associated feature of ACR in liver transplantation. The absence of peripheral eosinophilia predicted the absence of moderate/severe ACR, however it could not be used to predict or to assess the response to corticosteroids for the treatment of acute rejection. In a more recent study, based on 690 consecutive first liver transplant patients and using protocol liver biop- 
sies, peripheral eosinophil count was strongly associated with moderate-severe ACR. These investigators also found that the delta in eosinophil count between the biopsies performed before and after ACR treatment was the only independent predictor of histological improvement (Germani et al, 2015).

The role of IL-9, IL-23 and IL-17 in liver transplantation remains to be clarified. As far as IL-9 is concerned, when serum levels were determined in 50 liver transplanted patients (15 patients with ACR episodes, and 35 patients without ACR) on day $1 \& 7$ after liver transplantation and on the day of liver biopsy, with neither difference between patients nor ACR. The serum concentrations of IL-23 and IL-17 were not different early in the post-trans-plantation period. However, a significant increase in serum IL-23 levels in the ACR group was seen at the time of liver biopsy (Fábrega et al., 2009). The data were confirmed by a latter prospective study (Fan et al., 2012) showing that the levels of circulating CD4+IL-17+ T cells were higher in ACR patients than those without. The frequency of CD4+IL-17+ cells in peripheral blood correlated with ACR histological severity (Flores et al, 2016). Raschzok et al. (2015) postulated that CD44 can be used as a biomarker for the blood test-based diagnosis of rejection in liver transplant recipients. These results may help to identify patients at risk for ACR in first months after transplantation to improve clinical care the recipients.

\section{Conclusion}

No doubt, the liver transplantation (LT) has emerged as an established therapeutic option for patients with chronic liver disease worldwide.

There was correlation between different pathological findings in liver biopsy and serum level of CD44, lower levels being associated with rejection. CD44 may be used as a marker to detect acute liver rejection post transplantation. Prospective multicenter studies with longer follow up period and more patients will confirm and prove the update findings.

\section{References}

Adam, A, Asterisk, L, Karam, V, Delvart, V, et al, 2012: Evolution of indications and results of liver transplantation in Europe: A report from the European Liver Transplant Registry (ELTR). J. Hepatol. 57, 3:675-88.

Ahmed, OA, Kaisar, HH, Hawash, N, et al, 2017: Efficacy of sofosbuvir plus ribavirin with or without peginterferon-alfa in treatment of a cohort of Egyptian patients with hepatitis $\mathrm{C}$ virus infection. Infect. Disord. Drug Targets 17, 2:95-100.

Ahmed OA, Kaisar HH, Badawi R, et al, 2018: Efficacy and safety of sofosbuvir ledipasvir for treatment of a cohort of Egyptian patients with chronic hepatitis $\mathrm{C}$ genotype 4 infection. Infect Drug Resist. 11:295-8.

Abd-Elsalam, S, Sharaf-Eldin, M, Soliman, S, Elfert, A, Badawi, R, et al, 2018: Efficacy and safety of sofosbuvir plus ribavirin for treatment of cirrhotic patients with genotype 4 hepatitis C virus in real-life clinical practice. Arch. Virol. 163, 1:51-66.

Acevedo, J, Fernandez, J, 2014: New determnants of prognosis in bacterial infections in cirrhosis. World J. Gastroenterol. 20:7252-9.

Asaoka, T, Marubashi, S, Kobayashi, S, Hama, N, Eguchi, H, 2012: Intragraft transcriptome level of CXCL9 as biomarker of acute cellular rejection after liver transplantation. J. Surg. Res. 178, 2:1003-14

Brouard, S, Soulillou, JP, 2010: Pre-transplant serum level of CXCL9 as a biomarker of acute rejection and graft failure risk in kidney transplantation. Transpl. Int. 23:461-2.

Calne, RY, Williams, R, Dawson, JL et al, 1968: Liver transplantation in man. II. A report of two orthotopic liver transplants in adult recipients. Br. Med. J. 4, 5630:541-6.

Chen, R, Sigdel, TK, Li, L, Kambham, N, Dudley, JT, Hsieh SC, et al, 2010: Differential tially expressed RNA from public microarray data identifies serum protein biomarkers for data identifies serum protein biomarkers for crossorgan transplant rejection and other conditions. PLoS. Comput. Biol.6: e1000940.

Choudhary, NS, Saigal, S, Bansal, RK, Saraf, N, Gautam, D, 2017: Acute and Chronic Rejection After Liver Transplantation: What a clinician needs to know? J. Clin. Exp. Hepatol. doi: 10.1016/j.jceh.2017.10.003.

Demetris, A, Adams, D, Bellamy, C, Blakol- 
mer, K, Clouston, A, et al, 2000: Update of the International Banff Schema for liver allograft rejection: Working recommendations for the histopathologic staging and reporting of chronic rejection, Hepatology 31, 3:792-9.

Dutkowski, P, De Rougemont, O, Müllhaupt, B, Clavien, P, 2010: Current and future trends in liver transplantation in Europe. Gastroenterology 138, 3:802-9.

Fábrega, E, López-Hoyos, M, San Segundo, D, Casafont, F, et al, 2009: Changes in the serum levels of interleukin-17/interleukin-23 during acute rejection in liver transplantation. Liver Transplant. 15, 6:629-33.

Fan, H, Li, LX, Han, DD, Kou, JT, P. Li, p, et al, 2012: Increase of peripheral Th17 lymphocytes during acute cellular rejection in liver transplant recipients. Hepatobiliary Pancreat. Dis. Int. 15, 6:606-11.

Feussner, G, Stech, C, Dobmeyer, J, Schaefe, HR, Otto, G, et al, 1994: Serum amyloid A protein (SAA): a marker for liver allograft rejection in humans. Clin. Investig. 72, 12:1007-11.

Germani, G, Pleguezuelo, M, Villamil, F, Vaghjiani, SE, Tsochatziset, SE, et al, 2009: Azathioprine in liver transplantation: A reevaluation of its use and a comparison with mycophenolate mofetil. Am. J. Transplant. 9, 8:1725-31.

Germani, G, Castro, KR, Russo, FP, Senzolo, M, Zanetto, A, 2015: Markers of acute rejection and graft acceptance in liver transplantation. Wld. J. Gastroenterol. 21, 4:1061-8.

Graziadei, I, Zoller, H, Fickert, P, et al, 2016: Indications for liver transplantation in adults. Wien Klin. Wochenschr. 128:679-90.

Jalan, R, Gines, P, Olson, J, et al, 2012: Acute-on-chronic liver failure. J. Hepatol. 57:133648.

Katoh, S, McCarthy, JB, Kincade, PW, 1994: Characterization of soluble CD44 in the circulation of mice: Levels affected by immune activity and tumor growth. J. Immunol. 153:3440-9.

Kita, Y, Iwaki, Y, Demetris, AJ, Starzl, TE, 1994: Evaluation of sequential serum interleukin-6 levels in liver allograft recipients. Transplantation 57:1037-41.

Höroldt, BS, Burattin, M, Gunson, BK, Bramhall, SR, Nightingale, $P$, et al.2006: Does the Banff rejection activity index predict outcome in patients with early acute cellular rejection following liver transplantation? Liver Transplant. 12, 7:1144-51.

Lee, JG, 2014: How Can anti-thymocyte globu- lin be used in a liver transplant recipient? Rev. Immunosuppressants 12:347-348.

Massoud, O, Heimbach, J, Viker, K, et al, 2010: Noninvasive diagnosis of acute cellular rejection in liver transplant recipients: A proteomic signature validated by ELISA. Liver Transplant. 17, 6:723-32.

Flores, SM, Molina, GH, Enríquez, AB, FazMuñoz, D, Yeraldin Esquivel, Y, 2016: Cytokines and effector/ regulatory cells characterization in the physiopathology of cutaneous lupus erythematous: A cross-sectional study. Mediators Inflamm. 2016: 7074829. doi: 10.1155/707 4829

Moreau, R, Jalan, R, Gines, P, et al, 2013: Acute-on chronic liver failure is a distinct syndrome that develops in patients with acute decompensation of cirrhosis. Gastroenterology 144:1437.

Morris, MW, Anderson, C, 2013: The holygrail: A biomarker for acute rejection in liver transplantation. J. Surg. Res. 185, 2:535-6.

Raschzok, N, Reutzel-Selke, A, Schmuck, RB, Morgul, MH, Gauger, U, et al, 2015: CD44 and CXCL9 serum protein levels predict the risk of clinically significant allograft rejection after liver transplantation. Liver Transplant. 21, 9: 1195-207.

Roedder, S1, Vitalone, M, Khatri, P, Sarwal, MM, 2011: Biomarkers in solid organ transplantation: Establishing personalized transplantation medicine .Genome Med. 10.1186/gm253.

Rook, M, Rand, E, 2011: Predictors of longterm outcome after liver transplant. Curr. Opin. Organ Transplant. doi: 10.1097/ MOT.0b013e. 32834a945d.

Rostaing, L, Saliba, F, Calmus, Y, Dharancy, S, Boillot, O, 2012: Review article: Use of induction therapy in liver transplantation, Transplant. Rev. 26, 4:246-60.

Rouschop, KM, van den Beucken, T, Dubois, L, Niessen, H, Bussink, J, et al, 2010: The unfolded protein response protects human tumor cells during hypoxia through regulation of the autophagy genes MAP1LC3B and ATG5. J. Clin. Invest. 120, 1:127-41

Sagmeister, M, Mullhaupt, B, Kadry, Z, Kullak-Ublick, GA, Clavien PA, et al, 2002: Cost-effectiveness of cadaveric and living-donor liver transplantation. Transplantation 73:616-22. Sharaf-Eldin, M, Bediwy, AS, Kobtan, A, et al, 2016: Pigtail catheter: A less invasive option for pleural drainage in Egyptian patients with 
recurrent hepatic hydrothorax. Gastroenterol. Res. Pract. 2016:4013052

Sharma, A, 2017: CD44: A multifaceted molecule in cancer progression. J. Cancer Prev. Curr. Res. 1, 8:3-7.

Starzl, TE, Marchioro, TL, Porter, KL, Brettschneide, LR, 1967: Homotransplantation of the liver. Transplantation 5, 4:790-803.

Tilg, H1, Vogel, W, Aulitzky, WE, Herold, M,
Königsrainer, A, et al.1990: Evaluation of cytokines and cytokine-induced secondary messages in sera of patients after liver transplantation. Transplantation 49, 6:1074-80.

Yan, Y, Zuo, X, Wei, D, 2015: Cancer stem cells concise review: Emerging role of CD44 in cancer stem cells: A promising biomarker and therapeutic target. Stem Cells Transl. Med. 4, 9:1033-43. 